\title{
Irish Vegetation Database
}

\author{
Úna FitzPatrick \& Naomi Kingston
}

\begin{abstract}
The Irish Vegetation Database (GIVD ID EU-IE-001) was established in 2007 by the National Biodiversity Data Centre, in conjunction with the National Parks and Wildlife Service. It aims to collate all of the vegetation data that has been collected over the years in Ireland onto a single TURBOVEG database, and to make the maximum use possible of this valuable resource. In Ireland, the national vegetation database is already being used as an important national baseline. The historical data capture phase will be completed in 2012, when the database is expected to have in the region of 28,000 independent vegetation relevés. Ongoing surveys over the coming five years should increase this to between 28,000 and 32,000 relevés. Initially, the database will be used to create map layers showing the distribution of habitats and vegetation communities in Ireland. It will assist with reporting on implementation of the Habitats Directive (Article 17), and will support the research community in Ireland. The long term aims are to use the database to create a national vegetation classification system for Ireland; and to inform the development of a national vegetation and ecosystem map.
\end{abstract}

Keywords: baseline; Ireland; vegetation classification.

GIVD Database ID: EU-IE-001

Last update: $2012-05-06$

\section{Irish Vegetation Database}

Scope: The Irish Vegetation Database contains vegetation sampling units based on a percentage cover abundance scale, collected within the Republic of Ireland. Relevés collected using the DAFOR scale are not accepted into the database. For inclusion, relevés must have the following mandatory information: species list, cover abundance scale used, date, recorder, grid reference, and relevé size.

Status: ongoing capture Period: 1949-2011

Database manager(s): Una FitzPatrick (ufitzpatrick@biodiversityireland.ie); Naomi Kingston (naomi.kingston@ahg.gov.ie)

Owner: [NA]

Web address: http://nationalvegetationdatabase.biodiversityireland.ie

Availability: according to a specific agreement

Database format(s): TURBOVEG

Online upload: no

Online search: no

Publication: Weekes, L., Kingston, N. \& FitzPatrick Ú. (2012) Irish vegetation data in 2012, moving towards a national vegetation classification system. Irish Vegetation No. 1, National Parks and Wildlife Service, Department of Arts, Heritage and the Gaeltacht, Dublin, Ireland.

Plot type(s): normal plots

Plot-size range: $0.1-20 \mathrm{~m}^{2}$

Non-overlapping plots: 26,412

Estimate of existing plots: 28,000

Completeness: $94 \%$

Total plot observations: 26,412

Number of sources: 165

Valid taxa: 1,931

Countries: IE: $100.0 \%$

Forest: $15 \%$ - Non-forest: aquatic: 7\%; semi-aquatic: $23 \%$; arctic-alpine: $0 \%$; natural: $11 \%$; semi-natural: $42 \%$; anthropogenic: $2 \%$

Guilds: all vascular plants: $100 \%$; bryophytes (terricolous or aquatic): $35 \%$; lichens (terricolous or aquatic): $10 \%$; algae (terricolous or aquatic): $4 \%$ Environmental data: altitude: $30 \%$; slope aspect: $30 \%$; slope inclination: $30 \%$; soil depth: $20 \%$; surface cover other than plants (open soil, litter, bare rock etc.): $10 \%$; soil $\mathrm{pH}$ : $15 \%$; other soil attributes: $5 \%$; land use categories: $15 \%$

Performance measure(s): cover: $100 \%$

Geographic localisation: GPS coordinates (precision $25 \mathrm{~m}$ or less): $25 \%$; point coordinates less precise than GPS, up to $1 \mathrm{~km}$ : $60 \%$; small grid (not coarser than $10 \mathrm{~km}): 14 \%$; political units or only on a coarser scale $(>10 \mathrm{~km}): 1 \%$

Sampling periods: 1940-1949: 0.2\%; 1950-1959: 1.4\%; 1960-1969: 6.1\%; 1970-1979: 13.2\%; 1980-1989: 10.3\%; 1990-1999: 34.9\%; 2000-2009: 33.8\%; 2010-2019: 0.1\%

Information as of 2012-07-12; further details and future updates available from http://www.givd.info/ID/EU-IE-001

Úna FitzPatrick* (ufitzpatrick@biodiversityireland.ie)

National Biodiversity Data Centre, WIT West Campus, Carriganore Waterford, IRELAND

Naomi Kingston (naomi.kingston@ahg.gov.ie)

National Parks and Wildlife Service, Department of Arts, Heritage and the Gaeltacht, 7 Ely Place Dublin 2

*Corresponding author 